\title{
Adverse events following vaccination with an inactivated, Vero cell culture-derived Japanese encephalitis vaccine in the United States, 2009-2012
}

\author{
Ingrid B. Rabe ${ }^{\mathrm{a},{ }^{*}}$, Elaine R. Miller ${ }^{\mathrm{b}}$, Marc Fischer ${ }^{\mathrm{a}}$, and Susan L. Hills ${ }^{\mathrm{a}}$ \\ ${ }^{a}$ Arboviral Diseases Branch, Centers for Disease Control and Prevention, 3156 Rampart Road, \\ Fort Collins, CO 80521, United States \\ ' Immunization Safety Office, Centers for Disease Control and Prevention, 1600 Clifton Road NE, \\ Atlanta, GA 30333, United States
}

\section{Abstract}

Background-In March 2009, the U.S. Food and Drug Administration licensed an inactivated, Vero cell culture-derived Japanese encephalitis vaccine (JE-VC [Ixiaro]) for use in adults. The vaccine was licensed based on clinical trial safety data in $3558 \mathrm{JE}-\mathrm{VC}$ recipients. It is essential to monitor post-licensure surveillance data to evaluate the safety of JE-VC because rare adverse events may not be detected until the vaccine is administered to a larger population.

Methods-We reviewed adverse events reported to the U.S. Vaccine Adverse Event Reporting System (VAERS) for adults ( $\geq 17$ years) who received JE-VC from May 2009 through April 2012. Adverse event reporting rates were calculated using 275,848 JE-VC doses distributed.

Results-Over the 3 year period, 42 adverse events following vaccination with JE-VC were reported to VAERS for an overall reporting rate of 15.2 adverse events per 100,000 doses distributed. Of the 42 total reports, $5(12 \%)$ were classified as serious for a reporting rate of 1.8 per 100,000 doses distributed; there were no deaths. Hypersensitivity reactions $(N=12)$ were the most commonly reported type of adverse event, with a rate of 4.4 per 100,000 doses distributed; no cases of anaphylaxis were reported. Three adverse events of the central nervous system were reported (one case of encephalitis and two seizures) for a rate of 1.1 per 100,000; all occurred after receipt of JE-VC with other vaccines.

Conclusions-These post-marketing surveillance data suggest a good safety profile for JE-VC consistent with findings from pre-licensure clinical trials. Post-licensure safety data should continue to be monitored for any evidence of rare serious or neurologic adverse events.

\section{Keywords}

Japanese encephalitis vaccines; Vaccines; United States; Public health surveillance

*Corresponding author at: Arboviral Diseases Branch, Centers for Disease Control and Prevention, 3156 Rampart Road, Mail Stop P-02, Fort Collins, CO 80521, United States. Tel.: +1 970225 3245; fax: +1 970266 3568. irabe@cdc.gov (I.B. Rabe).

Conflict of interest statement

The authors have no relevant affiliations or financial involvement with any organization or entity with a financial interest in or financial conflict with the subject matter or materials discussed in the manuscript. No writing assistance was utilized in the production of this manuscript. 


\section{Introduction}

Japanese encephalitis (JE) virus, a mosquito-borne flavivirus, is the leading cause of vaccine-preventable encephalitis in Asia [1]. JE is a serious disease with a case-fatality rate of $20-30 \%$ and sequelae in $30-50 \%$ of survivors. The risk of JE for most travelers to Asia is very low but varies based on season, destination, duration, and activities [2]. The U.S. Advisory Committee on Immunization Practices (ACIP) recommends JE vaccine for travelers who plan to spend $\geq 1$ month in endemic areas during the JE virus transmission season; JE vaccine should be considered for short-term ( $<1$ month) travelers with higher risk itineraries [3].

In March 2009, the U.S. Food and Drug Administration (FDA) licensed an inactivated, Vero cell culture-derived Japanese encephalitis vaccine (JE-VC [Ixiaro]) for use in adults aged $\geq 17$ years [4]. JE-VC is administered in a 2-dose primary series at 0 and 28 days. If the primary series was administered $>1$ year previously, a booster dose may be given before potential JE virus exposure [5]. Prior to JE-VC becoming available, an inactivated mouse brain-derived JE vaccine (JE-MB [JE-VAX]) was the only JE vaccine licensed in the United States [3]. JE-MB was associated with rare but serious allergic and neurologic adverse events [6]. Allergic hypersensitivity reactions reported included generalized urticaria and angioedema of the face, oropharynx, and extremities; neurologic events included acute disseminated encephalomyelitis temporally-associated with vaccination [2]. In 2006, the manufacturer ceased production of JE-MB and all supplies expired by May 2011 [7].

JE-VC was licensed based on clinical trial safety data in $3558 \mathrm{JE}-\mathrm{VC}$ recipients. No important safety concerns were identified [8-10]. However, it is essential to monitor postlicensure surveillance data to evaluate the safety of JE-VC in a larger population [3]. We analyzed adverse events reported to the U.S. Vaccine Adverse Event Reporting System (VAERS) in adults who received JE-VC during the first 3 years post-licensure. Based on the adverse event profile of the previously-available JE-MB, we focused on serious, hypersensitivity, and neurologic adverse events.

\section{Methods}

\subsection{VAERS}

VAERS is a national passive surveillance system for monitoring adverse events following immunization [11]. VAERS is co-administered by the U.S. Centers for Disease Control and Prevention (CDC) and FDA. Vaccine manufacturers, healthcare providers, and vaccine recipients submit reports online, by fax, or by mail, using a standardized form that includes data on patient demographics, vaccination, and adverse events. VAERS administrators assign Medical Dictionary for Regulatory Activities (MedDRA) codes to the reported adverse events. For individual VAERS reports, the causal relationship between vaccination and reported events cannot usually be determined. 


\subsection{Case definitions and classification}

We reviewed reports of adverse events received by VAERS as of May 1, 2013 following administration of JE-VC to adults ( $\geq 17$ years) from May 1, 2009 through April 30, 2012, either in the United States or to U.S. military personnel stationed abroad. We defined onset interval as the number of days from vaccination (Day 0 ) until the first reported symptom. Reports were excluded if the onset interval was $>60$ days $(N=2$, including one report of anaphylaxis 67 days after receipt of JE-VC and one report of acute myelogenous leukemia with onset 110 days after receipt of JE-VC), or if the only event that occurred was a local reaction in the arm contralateral to where JE-VC was administered $(N=5)$. Reports were also excluded if they described errors in vaccine administration but no adverse event (i.e., administration of JE-VC during pregnancy $[N=1]$ and administration of expired vaccine $[N$ $=1]$ ). Additionally, one report was excluded because a retrospective record review confirmed that the report was based on a disease coding error and there was no adverse event; another report was excluded because it listed only the coding term "convulsion" but contained no data on patient demographics, date of vaccine receipt, or date of adverse event onset.

A serious adverse event was defined according to the FDA regulatory definition (21 CFR 600.80) as life-threatening or resulting in death, inpatient hospitalization or prolongation of existing hospitalization, persistent or significant disability, or a congenital anomaly/birth defect [12]. Adverse events were classified as anaphylaxis if they met level 1 or level 2 of diagnostic certainty using the Brighton Collaboration case definition, and occurred within 2 $h$ of vaccination [13]. An adverse event report was classified as a hypersensitivity reaction if any of the major or minor dermatologic/mucosal or respiratory criteria of the Brighton Collaboration case definition for anaphylaxis were present and occurred within 14 days of vaccination $[6,13]$. Hypersensitivity reactions were classified as "immediate" if onset interval was $<2 \mathrm{~h}$ and "delayed" if onset interval was $2 \mathrm{~h}$ to 14 days after vaccination. Adverse events classified as central neurologic events included aseptic meningitis, encephalitis, myelitis, acute disseminated encephalomyelitis (ADEM), Guillain-Barré syndrome (GBS), or generalized seizure that met level 1 or level 2 of diagnostic certainty of the Brighton Collaboration case definitions for those conditions [14-17]. Adverse events were classified as peripheral neurologic adverse events if they were confined to the peripheral nervous system (e.g., paresthesia and peripheral neuritis).

\subsection{Data collection and analysis}

We reviewed VAERS reports for patient age, sex, concurrent vaccine administration, onset interval, MedDRA coding terms, symptom text, and outcome. Medical records, which are routinely obtained for serious adverse events, were reviewed. Starting in August 2010, we also contacted the vaccine recipient or healthcare provider to obtain additional clinical data on reports of serious, hypersensitivity, or neurologic reactions. For reports which included multiple coding terms, the key event was determined. Because VAERS is a routine surveillance program conducted as a public health function, it is not subject to Institutional Review Board review and informed consent requirements. 
The analysis was conducted by unique case report. The incidence of all, serious, hypersensitivity, and neurologic adverse events reported following vaccination with JE-VC were calculated per 100,000 doses distributed during the 3 year period. Based on data obtained from Valneva Austria GmbH (formerly Intercell Biomedical) and Novartis Vaccines, 275,848 JE-VC doses were distributed to the U.S. private market and military from May 2009 through April 2012. No information was available regarding the numbers of vaccine doses administered or the age and sex of vaccine recipients.

\section{Results}

\subsection{Adverse event reports following receipt of JE-VC}

During the 3 year period, 42 adverse events following receipt of JE-VC were reported to VAERS and met the inclusion criteria for an overall reporting rate of 15.2 adverse events per 100,000 doses distributed (Table 1). Twenty-two (52\%) of the reported events occurred in males and the median age was 31 years (range 19-59 years) (Table 2). Adverse events occurred a median of 1 day after vaccination (range 0-39 days); 38 (90\%) occurred within 2 weeks of vaccination. Twenty-seven (64\%) events occurred after the first dose of JE-VC and $10(24 \%)$ followed a second dose.

JE-VC was listed as the only vaccine administered in 17 (40\%) of the 42 reports. Among the other 25 reports, a median of two vaccines (range 1-5) was administered at the same time as JE-VC. The most common concurrently administered vaccines were typhoid $(N=8)$, hepatitis A $(N=7)$, hepatitis B $(N=6)$, rabies $(N=6)$, anthrax $(N=5)$, tetanus/diphtheria/ acellular pertussis (Tdap) $(N=4)$, smallpox $(N=3)$, and influenza $(N=2)$. There were no substantial differences in the median age, sex, or onset intervals between events that occurred following JE vaccine administered alone or with other vaccines (Table 3).

\subsection{Classification of adverse events}

Of the 42 adverse events reported, 5 (12\%) were classified as serious and $37(88 \%)$ as nonserious; $12(29 \%)$ reports were classified as hypersensitivity reactions and three $(7 \%)$ as central neurologic events. The 22 reports that did not meet the case definition for serious, neurologic, or hypersensitivity adverse events, included fever $(N=10)$, musculoskeletal pain $(N=3)$, local reactions $(N=3)$, nonspecific somatosensory complaints $(N=3)$, rashes $(N=$ $2)$, and syncope $(N=1)$.

3.2.1. Serious adverse events-Five (12\%) adverse events were classified as serious for a reporting rate of 1.8 per 100,000 doses distributed (Table 1). These occurred in males with a median age of 40 years (range 20-56) (Table 2). Three serious events occurred following a first dose, one following a second dose, and one report did not include the dose number. Four of the patients with serious adverse events were hospitalized and one was observed for a "life-threatening illness" but not hospitalized. There were no deaths.

JE-VC was the only vaccine administered for two of the reports (Table 4). One was a delayed hypersensitivity event and the other was a patient who developed abdominal pain at 5 days post-vaccination and had an appendectomy for appendicitis. The other three serious adverse events were reported following administration of JE-VC with other vaccines. One 
was classified as an immediate hypersensitivity reaction and one as a neurologic event (encephalomyelitis). The remaining serious report was a patient who developed chest pain at 11 days after receiving JE-VC, smallpox, anthrax, and typhoid vaccines and was diagnosed with myopericarditis. He was hospitalized for 3 days and discharged after clinical improvement.

3.2.2. Hypersensitivity reactions-Overall, 12 (29\%) reports were classified as hypersensitivity reactions, for a rate of 4.4 per 100,000 doses distributed (Table 1). Six (50\%) occurred in males and the median age of patients was 33 years (range 19-59). Seven $(58 \%)$ occurred following a first dose of JE-VC and five (42\%) following a second dose. Seven $(58 \%)$ occurred after vaccination with JE-VC alone and five (42\%) after concomitant administration of JE-VC with other vaccines (Table 4). Ten (83\%) events had onset $\unlhd$ day after vaccination, one at 2 days after vaccination, and one at 10 days after vaccination. Four (33\%) were immediate ( $<2 \mathrm{~h}$ after vaccination) and eight (67\%) were delayed hypersensitivity. Two hypersensitivity reactions (one immediate and one delayed) were classified as serious adverse events. No reports met the case definition for anaphylaxis.

Of the four immediate hypersensitivity reactions, two occurred in males and the median age of patients was 26 years (ranged 19-45). Onset ranged from 5 to $90 \mathrm{~min}$ after vaccination (median $13 \mathrm{~min}$ ). Two occurred after administration of JE-VC alone, one after a first dose, and one after a second dose. Two occurred after administration of JE-VC with other vaccines, both following a first dose of JE-VC. Three patients had dyspnea and a sensation of throat closure and one had generalized urticaria. None had cardiovascular symptoms and none were treated with epinephrine; however, all received diphenhydramine.

The eight delayed hypersensitivity events occurred equally among males and females with a median age of 45 years (range 21-59 years). The onset interval ranged from $2 \mathrm{~h}$ to 10 days (median 1 day); 7 (88\%) occurred within 3 days following vaccination. One report documented generalized urticaria with onset 10 days after the subject received JE-VC, smallpox, and anthrax vaccines; however, he also completed a 10-day course of oral amoxicillin 1 day prior to onset. Four events (50\%) followed a first dose of JE-VC and five events $(63 \%)$ occurred after vaccination with JE-VC alone. Overall, clinical findings included generalized urticaria $(N=4)$, generalized pruritic maculopapular rash $(N=1)$, localized pruritic rash $(N=1)$, dyspnea and cough $(N=1)$, and sensation of tongue swelling and numbness $(N=1)$. The patient who had sensation of tongue swelling and numbness after receiving JE-VC, hepatitis B, rabies, and typhoid vaccines, reported experiencing a similar event when she received JE-MB approximately 9 years earlier.

3.2.3. Neurologic events-One reported adverse event met the case definition for encephalomyelitis and two for seizures. There were no reports of aseptic meningitis, GBS, or ADEM following immunization with JE-VC vaccine. The three central neurologic adverse event reports constitute a reporting rate of 1.1 per 100,000 doses distributed.

The case of encephalomyelitis occurred in a 20-year-old male soldier who had onset of headache, altered mental status, photophobia, upper motor neuron signs, and pleocytosis at 39 days after receiving his first dose of JE-VC, which was administered concomitantly with 
oral typhoid, smallpox, and hepatitis A/hepatitis B vaccines. Five days before his onset of symptoms he received another dose of smallpox vaccine because of failure to develop an appropriate immune response to the previous dose. He was hospitalized for 2 days and recovered with no complications.

One seizure report described loss of consciousness and abnormal limb movements in a 35year-old female with underlying neuropsychiatric conditions. The event was witnessed by medical staff and occurred immediately after the patient received JE-VC, Tdap, hepatitis A, and rabies vaccines. The other seizure report described loss of consciousness, jerking limb movements, and vomiting in a 30-year-old female at $8 \mathrm{~h}$ after receiving JE-VC and rabies vaccines; the event was witnessed by friends at home. This patient had also ingested a homeopathic preparation and cannabis on the same day. She was medically evaluated as an outpatient in the subsequent week.

There were also three reports of peripheral neurologic adverse events. These included one report of upper limb neuritis in the arm where JE-VC was administered (with hepatitis A/ hepatitis $\mathrm{B}$ vaccine administered in the contralateral arm), one report of paresthesia in the limb where JE-VC was administered with meningococcal vaccine, and one report of generalized paresthesia following administration of JE-VC with hepatitis A and varicella vaccines.

\section{Discussion}

A total of 42 adverse events following receipt of JE-VC vaccine were reported to VAERS during the first 3 years post-licensure when over 275,000 doses were distributed. The most frequently reported events were hypersensitivity reactions and fever. Most events were nonserious, no unexpectedly high reporting rates for specific events were identified, and no deaths were reported. Although the possibility of rare serious adverse events associated with this vaccine cannot be excluded, these post-marketing data support the safety profile of JE$\mathrm{VC}$ from pre-licensure clinical trials.

The overall rate of 15.2 adverse events per 100,000 doses distributed is similar to the rate reported to VAERS for JE-MB from 1993-1999 (15.0 per 100,000 doses distributed) and lower than that reported for JE-MB from 1999-2009 (23.7 per 100,000 doses) [6,18]. The reporting rate for serious adverse events following JE-VC was equivalent to the rate detected following JE-MB (1.8 per 100,000 doses), and may reflect concomitant administration of JE-VC with other reactogenic vaccines [6]. From 2009 to 2012, three (60\%) of the five reported serious adverse events occurred following receipt of JE-VC with other vaccines. These included one report each of myopericarditis and encephalomyelitis in patients who had also received smallpox vaccine. Myocarditis is a well-described complication following smallpox vaccination; encephalitis has also been reported [19].

Hypersensitivity reactions were reported in 12 recipients; most included primarily dermatological symptoms and none met the case definition for anaphylaxis. Eleven of the reactions occurred within 2 days after vaccination. Other etiologies for such reactions cannot be ruled out, but the temporal association may support a causal association with JE-VC 
vaccination, especially for the seven hypersensitivity reactions that occurred among persons vaccinated with JE-VC alone. Gelatin, used as a vaccine stabilizer in JE-MB and considered a possible cause of the allergic hypersensitivity reactions occurring following JE-MB, is not present in JE-VC, leading to the assumption that JE-VC might be associated with fewer hypersensitivity events [20]. In this analysis, the reporting rate for hypersensitivity reactions following JE-VC was 4.4 per 100,000 doses distributed compared with 6.3-8.4 per 100,000 doses following JE-MB $[6,18]$.

We identified one report of hypersensitivity following JE-VC in a patient who experienced similar symptoms following previous vaccination with JE-MB. A recent case report describing a patient who had a hypersensitivity reaction following a dose of JE-MB and again following a dose of JE-VC administered 3 years later suggested that a common factor in both vaccines may be responsible, possibly JE virus antigen [21]. However, any conclusions are limited by the small numbers and inability to determine causality in these reports.

Three central neurologic events following JE-VC were reported, for a rate of 1.1 per 100,000 , compared with 0.3 per 100, 000 doses distributed following JE-MB. However, these events occurred after JE-VC administered on the same day as other vaccines.

Furthermore, the encephalomyelitis case occurred 39 days after JE-VC vaccination and only 5 days after a repeat smallpox vaccination. Similarly, the two reports of seizures occurred in patients with possible alternative etiologies. For JE-MB, concerns were raised regarding the potential for neurologic adverse effects because of murine neural proteins introduced through mouse brain passage. The production and formulation of JE-VC does not pose the same concerns $[3,8-10]$.

Pre-licensure clinical trials of JE-VC did not identify any important safety concerns or specific events that required post-surveillance monitoring [8-10,22]. The vaccine manufacturer evaluated post-marketing surveillance data from the first 12 months (April 2009-March 2010) following licensure in Europe, Australia, and the United States [22]. That analysis showed a lower rate of all adverse events (10.1 per 100,000 doses distributed) than the VAERS data from 2009 to 2012 reported here. This variation in findings may be due to differences in the study populations, surveillance systems, reporting criteria, or case classification. In addition, the proportion of distributed doses that are administered or awareness about the vaccine and adverse events may have changed over time. Importantly, the current and previous analyses showed similar reporting rates per 100,000 doses distributed for both serious adverse events (1.8 and 1.6) and hypersensitivity reactions (4.4 and 4.5 per 100,000$)$.

This report is subject to several limitations. VAERS is a passive surveillance system dependent on clinician or patient recognition and reporting of events [11]. The temporal association of an adverse event with vaccination does not prove a causal relationship. The lack of data on the number of doses administered versus distributed precludes the calculation of the true incidence of adverse events. From 1993-2009, >2 million doses of JE-MB were distributed, compared to $<300,000$ doses of JE-VC distributed from 2009 to 2012; this difference in numbers of doses distributed and any differences in reporting to VAERS over 
the analysis periods limits the ability to accurately compare rates of adverse events for JEVC and JE-MB. The relatively low number of JE-VC doses distributed over the reporting period may have precluded detection of rare adverse events.

\section{Conclusions}

The risk of travel-associated JE is low. However, there is no effective treatment, case fatality is high, and sequelae among survivors is substantial. Therefore, travelers with itineraries that place them at higher risk of infection should receive JE vaccine. These post-marketing surveillance data from the first 3 years post-licensure support the good safety profile documented in pre-licensure clinical trials. The apparent higher rate of central neurologic adverse events following JE-VC compared to JE-MB is likely artifactual but requires continued monitoring. In May 2013, FDA licensed JE-VC for use in children aged 2 months through 16 years and use in this age group was subsequently approved by the ACIP. Postmarketing surveillance safety data should continue to be monitored for any evidence of rare serious or neurologic adverse events in adults or children. Providers should report clinically significant adverse events following JE-VC to VAERS (www.vaers.hhs.gov).

\section{Acknowledgments}

We thank the VAERS staff for their data collection efforts, clinicians and patients for their willingness to provide information to complete the questionnaires, Katherine Gibney for development of the follow-up form, Katrin Dubischar-Kastner and Hayley Hughes for assistance in clarifying report data, and Nicole Lindsey, Jim Sejvar, and Erin Staples for assistance with case classification.

\section{References}

1. Campbell GL, Hills SL, Fischer M, Jacobson JA, Hoke CH, Hombach JM, et al. Estimated global incidence of Japanese encephalitis: a systematic review. Bull World Health Organ. 2011; 89(10): 766-74. [PubMed: 22084515]

2. Hills SL, Griggs AC, Fischer M. Japanese encephalitis in travelers from non-endemic countries, 1973-2008. Am J Trop Med. 2010; 82(5):930-6.

3. Centers for Disease Control and Prevention. Japanese encephalitis vaccines: recommendations of the Advisory Committee on Immunization Practices (ACIP). MMWR Recomm Rep. 2010; 59(RR-1): $1-27$.

4. United States Food and Drug Administration. [accessed 04.03.14] Ixiaro - summary basis for regulatory action. 2009. Available at: http://www.fda.gov/biologicsbloodvaccines/vaccines/ approvedproducts/ucm 142580

5. Centers for Disease Control and Prevention. Recommendations for use of a booster dose of inactivated vero cell culture-derived Japanese encephalitis vaccine: advisory committee on immunization practices, 2011. MMWR. 2011; 60(20):661-3. [PubMed: 21617632]

6. Lindsey NP, Staples JE, Jones JF, Sejvar JJ, Griggs A, Iskander J, et al. Adverse event reports following Japanese encephalitis vaccination in the United States, 1999-2009. Vaccine. 2010; 29(1): 58-64. [PubMed: 20970488]

7. Centers for Disease Control and Prevention. Update on Japanese encephalitis vaccine for children: United States, May 2011. MMWR. 2011; 60(20):664-5. [PubMed: 21617633]

8. Tauber E, Kollaritsch H, von Sonnenburg F, Lademann M, Jilma B, Firbas C, et al. Randomized, double-blind, placebo-controlled phase 3 trial of the safety and tolerability of IC51, an inactivated Japanese encephalitis vaccine. J Infect Dis. 2008; 198(4):493-9. [PubMed: 18588481]

9. Tauber E, Kollaritsch H, Korinek M, Rendi-Wagner P, Jilma B, Firbas C, et al. Safety and immunogenicity of a Vero-cell-derived, inactivated Japanese encephalitis vaccine: a non-inferiority, phase III, randomised controlled trial. Lancet. 2007; 370(9602):1847-53. [PubMed: 18061060] 
10. Dubischar-Kastner K, Kaltenboeck A, Klingler A, Jilma B, Schuller E. Safety analysis of a Verocell culture derived Japanese encephalitis vaccine, IXIARO (IC51), in 6 months of follow-up. Vaccine. 2010; 28(39):6463-9. [PubMed: 20673824]

11. Varricchio F, Iskander J, Destefano F, Ball R, Pless R, Braun MM, et al. Understanding vaccine safety information from the Vaccine Adverse Event Reporting System. Pediatr Infect Dis J. 2004; 26(5-6):575-85.

12. United States Food and Drug Administration. [accessed 04.03.14] Code of Federal Regulations Title 21, Sec. 600.80 Postmarketing reporting of adverse experiences. 2013. Available at: http:// www.accessdata.fda.gov/scripts/cdrh/cfdocs/cfcfr/cfrsearch.cfm?fr=600.80

13. Ruggeberg JU, Gold MS, Bayas JM, Blum MD, Bonhoeffer J, Friedlander S, et al. Anaphylaxis: case definition and guidelines for data collection, analysis, and presentation of immunization safety data. Vaccine. 2007; 25(31):5675-84. [PubMed: 17448577]

14. Tapiainen T, Prevots R, Izurieta HS, Abramson J, Bilynsky R, Bonhoeffer J, et al. Aseptic meningitis: case definition and guidelines for collection, analysis and presentation of immunization safety data. Vaccine. 2007; 25(31):5793-802. [PubMed: 17574313]

15. Sejvar JJ, Kohl KS, Bilynsky R, Blumberg D, Cvetkovich T, Galama J, et al. Encephalitis, myelitis, and acute disseminated encephalomyelitis (ADEM): case definitions and guidelines for collection, analysis, and presentation of immunization safety data. Vaccine. 2007; 25(31):5771-92. [PubMed: 17570566]

16. Sejvar JJ, Kohl KS, Gidudu J, Amato A, Bakshi N, Baxter R, et al. Guillain-Barre syndrome and Fisher syndrome: case definitions and guidelines for collection, analysis, and presentation of immunization safety data. Vaccine. 2011; 29(3):599-612. [PubMed: 20600491]

17. Bonhoeffer J, Menkes J, Gold MS, de Souza-Brito G, Fisher MC, Halsey N, et al. Generalized convulsive seizure as an adverse event following immunization: case definition and guidelines for data collection, analysis, and presentation. Vaccine. 2004; 22(5-6):557-62. [PubMed: 14741144]

18. Takahashi H, Pool V, Tsai TF, Chen RT. Adverse events after Japanese encephalitis vaccination: review of post-marketing surveillance data from Japan and the United States. The VAERS Working Group. Vaccine. 2000; 18(26):2963-9. [PubMed: 10825597]

19. Food and Drug Administration. ACAM2000 (Smallpox (Vaccinia) Vaccine, Live). Sanofi Pasteur Biologics Co; Product approval information [package insert]. Available at: http://www.fda.gov/ downloads/BiologicsBloodVaccines/Vaccines/ApprovedProducts/UCM142572.pdf [accessed 07.1114]

20. Sakaguchi M, Yoshida M, Kuroda W, Harayama O, Matsunaga Y, Inouye S. Systemic immediatetype reactions to gelatin included in Japanese encephalitis vaccines. Vaccine. 1997; 15(2):121-2. [PubMed: 9066026]

21. McCallum AD, Jones ME. Allergy to IXIARO and BIKEN Japanese encephalitis vaccines. J Travel Med. 2013; 20(1):60-2. [PubMed: 23279235]

22. Schuller E, Klingler A, Dubischar-Kastner K, Dewasthaly S, Muller Z. Safety pro-file of the Vero cell-derived Japanese encephalitis virus (JEV) vaccine Ixiaro. Vaccine. 2011; 29:8669-76.

[PubMed: 21907747] 


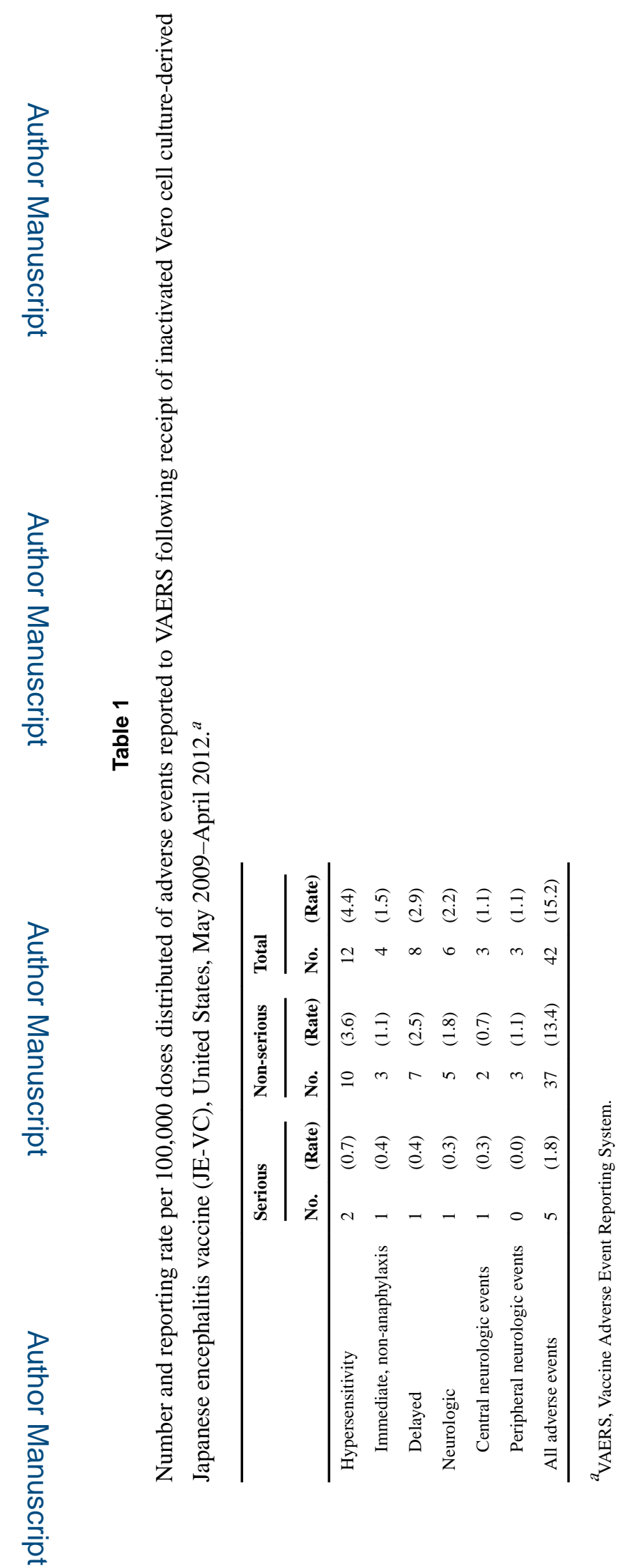

Vaccine. Author manuscript; available in PMC 2018 January 12. 


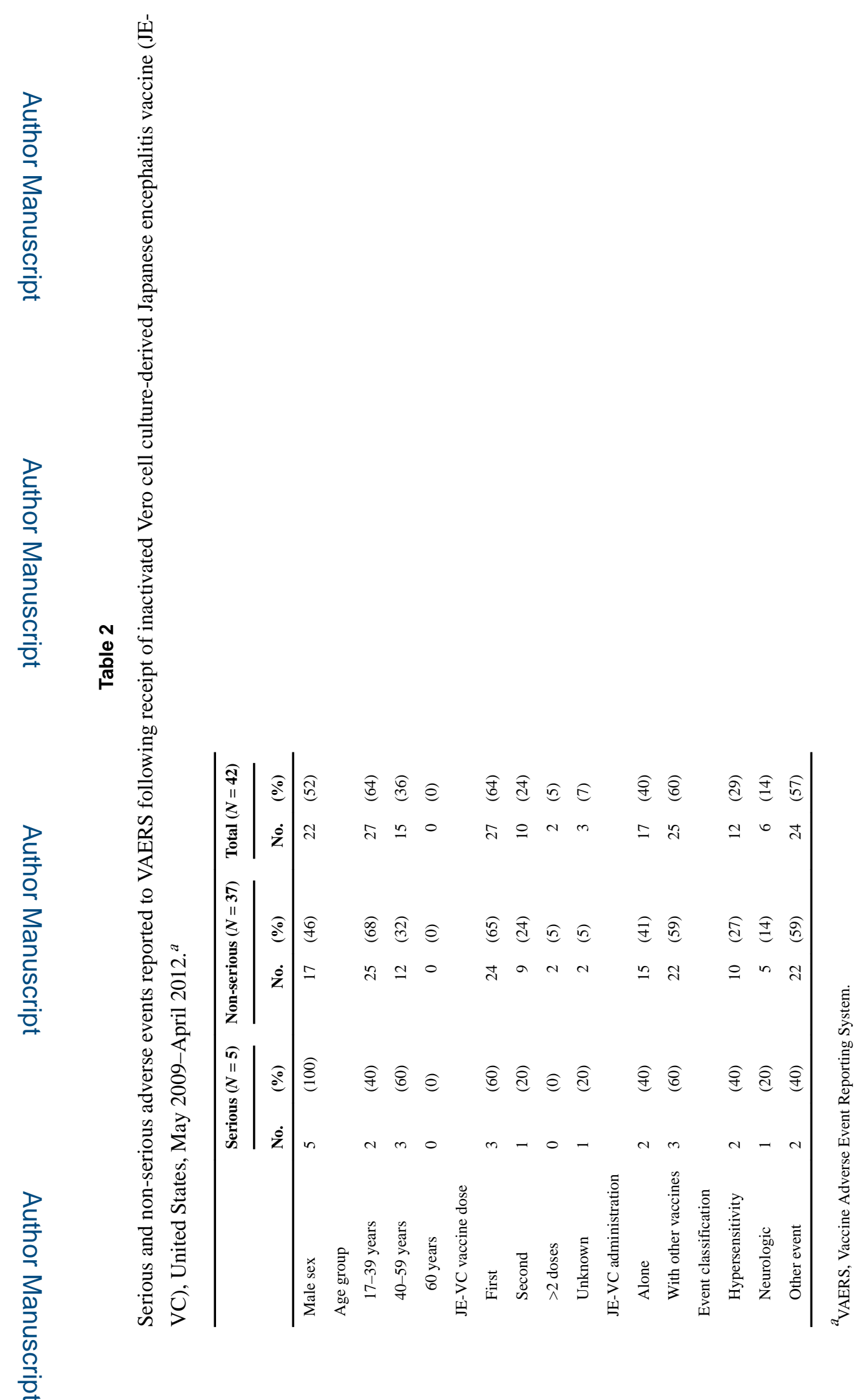




\section{Table 3}

Adverse events reported to VAERS following receipt of inactivated Vero cell culture-derived Japanese encephalitis vaccine (JE-VC) when administered alone or with other vaccines, United States, May 2009-April $2012 .^{a}$

\begin{tabular}{|c|c|c|c|c|}
\hline & \multicolumn{2}{|c|}{ JE-VC administered alone $(N=17)$} & \multicolumn{2}{|c|}{ JE-VC administered with other vaccines $(N=25)$} \\
\hline & No. & $(\%)$ & No. & $(\%)$ \\
\hline Male sex & 7 & $(41)$ & 10 & $(40)$ \\
\hline Serious adverse events & 2 & (12) & 3 & (11) \\
\hline \multicolumn{5}{|l|}{ JE-VC vaccine dose } \\
\hline First & 8 & (47) & 20 & $(80)$ \\
\hline Second & 6 & (35) & 4 & (16) \\
\hline$>2$ doses & 2 & (12) & 0 & $(0)$ \\
\hline Unknown & 1 & (6) & 1 & (4) \\
\hline \multicolumn{5}{|l|}{ Event classification } \\
\hline Hypersensitivity & 7 & $(41)$ & 5 & (19) \\
\hline Neurologic & 0 & (0) & 6 & $(11)$ \\
\hline Other event & 10 & (59) & 14 & (70) \\
\hline
\end{tabular}

${ }^{a}$ VAERS, Vaccine Adverse Event Reporting System. 


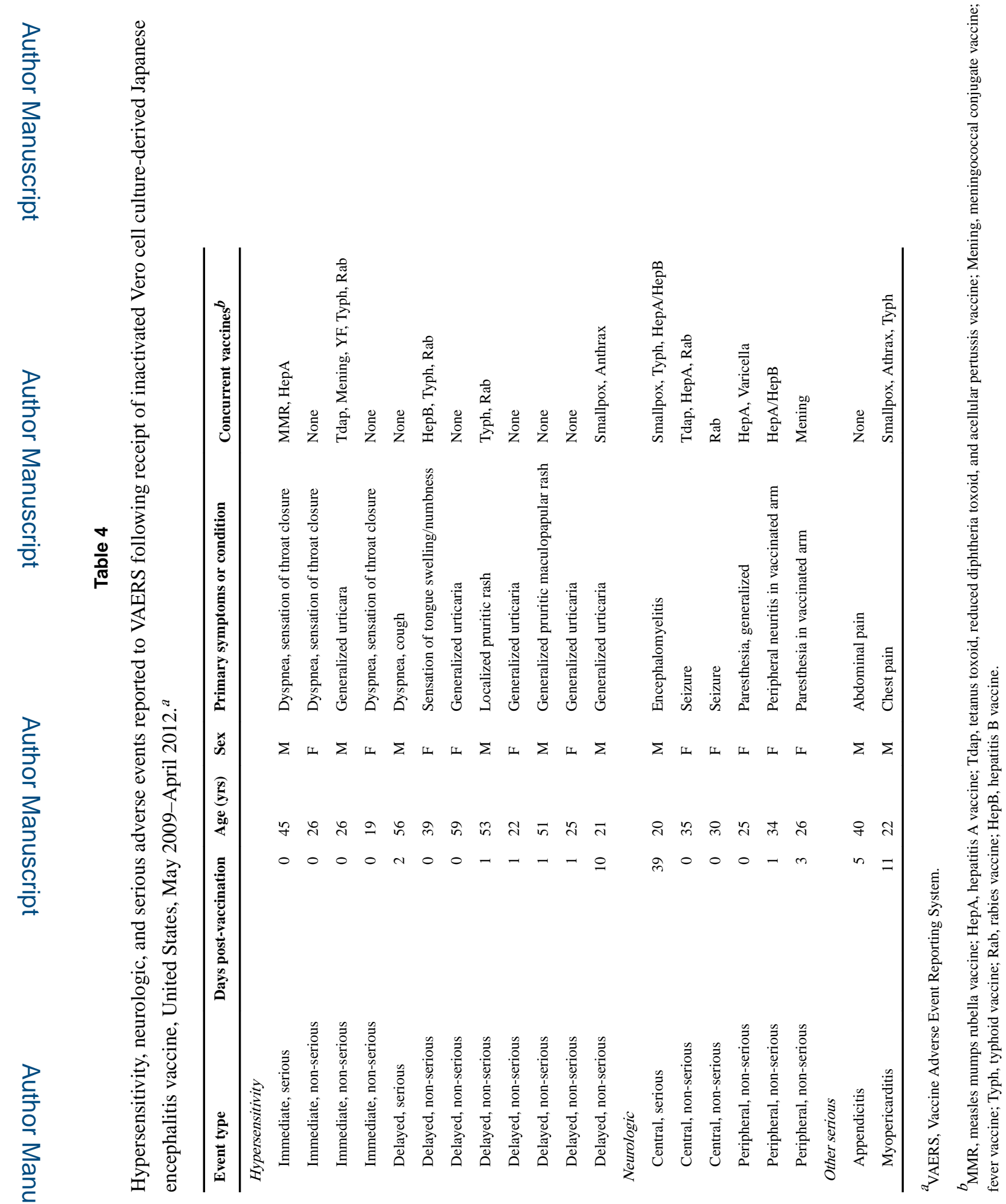

